\title{
Extrarenal calyces mimicking retroperitoneal cystic mass with concomitant ureteropelvic junction obstruction: renal pelvis reconstruction using calyx unification
}

This article was published in the following Dove Press journal:

Research and Reports in Urology

\author{
Irfan Wahyudi' \\ Arry Rodjani' \\ Gerhard Reinaldi Situmorang' \\ Prahara Yuri $^{2}$ \\ Fakhri Rahman' \\ 'Urology Department, Faculty of \\ Medicine, Universitas Indonesia, Cipto \\ Mangunkusumo Hospital, Jakarta, \\ Indonesia; ${ }^{2}$ Urology Department, Faculty \\ of Medicine, Universitas Gadjah Mada, \\ Sardjito Hospital, Yogyakarta, Indonesia
}

\begin{abstract}
Extrarenal calyces (ERCs) are a very rare urological anomaly, especially when concomitantly presenting with ureteropelvic junction obstruction (UPJO). Surgical intervention is often necessary in ERCs associated with UPJO, with dismembered pyeloplasty being the most commonly utilized technique. We present a case of UPJO-associated ERCs in which renal pelvis reconstruction using calyx unification was selected as the treatment technique. An 11-year-old boy was referred to our center due to bilateral hydronephrosis and left multicystic kidney disease. Magnetic resonance imaging showed severe left hydronephrosis with concomitant left cystic mass and left UPJO. A ${ }^{99 \mathrm{~m}} \mathrm{Tc}$ diethylenetriaminepentaacetic diuretic renal scan showed residual renal function of $25.9 \%$ split function and $26.8 \mathrm{~mL} /$ minute glomerular filtration rate. Intraoperatively, we found ERCs with severely dilated renal pelvis. The renal pelvis was excised. Major calyces protruding from the kidney were unified using side-to-side anastomosis to form a new structure resembling a renal pelvis, which was further anastomosed to the ureter. Temporary urinary drainage from the affected kidney was achieved using a double-J (DJ) stent and nephrostomy. Pathological examination revealed atrophic transitional epithelial cells. There was no intra- or postoperative complication reported. The nephrostomy tube and DJ stent were removed 2 weeks and 3 months after surgery, respectively. Ultrasonography examination performed at 1 and 9 months after DJstent removal showed no hydronephrosis. We conclude that renal pelvis reconstruction using calyx unification can be performed safely and is effective in treating patients with ERCs associated with PUJO. This technique should be considered especially in cases where excision of the renal pelvis cannot be avoided.
\end{abstract}

Keywords: extrarenal calyces, hydronephrosis, reconstructive surgical procedure, ureteropelvic junction obstruction, UPJO

\section{Introduction}

First described in 1925, extrarenal calyces (ERCs) are considered one of the rarest urinary tract anomalies, with $<60$ cases reported to date. ${ }^{1}$ This anomaly is often presented as an intraoperative surprise, due to the lack of accurate diagnostic tool. It is not uncommon for ERCs to be found along with other urinary tract anomalies, such as horseshoe kidney, ectopic kidney, renal dysplasia, and ureteropelvic junction obstruction (UPJO). ${ }^{2-5}$ Several studies have reported ERC cases that were associated with UPJO. The management of these cases varies depending on existing
Situmorang

Cipto Mangunkusumo Hospital, Faculty of Medicine, Universitas Indonesia, Jalan

Diponegoro No. 7I, Kenari, Senen, Jakarta 10430, Indonesia

Tel +62 21 3152892

Fax +62213145592

Email gr.situmorang@gmail.com 
residual renal function. ${ }^{4,6-8}$ When kidney function was preserved, dismembered pyeloplasty was the treatment of choice documented by most reports. ${ }^{7,8}$ To our knowledge, there has been no report on the use of renal pelvis reconstruction using calyx unification as a surgical technique for the treatment of ERCs with coexisting UPJO. This report presents a novel alternative approach in the management of UPJO-associated ERCs.

\section{Case}

An 11-year-old, male was referred to our department for evaluation of bilateral hydronephrosis and left multi-ystic kidney, which had been identified by ultrasonography (Figure 1A). The patient complained of left abdominal pain since 3 years before admission. He had hesitated to seek medical treatment, until the abdominal growth was markedly felt in the last year. He denied the presence of fever. Urinalysis revealed urinary tract infection. Other laboratory examinations showed normal results. Abdominal and pelvic magnetic resonance imaging showed left severe hydronephrosis with concomitant left cystic mass and left UPJO (Figure 1B). The dilated left kidney also compressed the right distal ureter, resulting in moderate right hydronephrosis. Percutaneous nephrostomy was placed into the left kidney. A ${ }^{99 \mathrm{~m}} \mathrm{Tc}$ diethylenetriaminepentaacetic acid (DTPA) diuretic renal scan showed severe decline in function of the left kidney $(25.9 \%$ split function and $26.8 \mathrm{~mL} /$ minute glomerular filtration rate [GFR]) and partial obstruction on the right kidney (76.9 $\mathrm{mL} /$ minute GFR). Daily production of the left nephrostomy tube was $100-150 \mathrm{~mL}$ urine. An open exploration of the left kidney followed by left pyeloplasty was planned. However, due to a personal problem of the patient, the surgery was performed 4 months after the initial ${ }^{99 \mathrm{~m}}$ Tc DTPA diuretic renal scan.
Open surgery was performed in the lumbotomy position. The left kidney was approached through an intercostal incision made between the 11th and 12th ribs. Cystic mass was identified after the Gerota fascia had been opened. The cystic mass was decompressed, and approximately $3 \mathrm{~L}$ fluid was drained. Evaluation after decompression revealed six ureter-like structures (major calyces) that arose from the left-kidney parenchyma, all of which were drained into the already-decompressed cystic mass, which was identified as the dilated renal pelvis (Figure 2). A normal ureter was identified, which connected the dilated renal pelvis to the urinary bladder. We decided to excise the massively dilated renal pelvis and perform renal pelvis reconstruction by unifying all ERCs identified. All six major calyces were spatulated and unified using side-to -side anastomosis between each calyx with continuous suture of polydioxanone 5-0 sutures. Subsequently, anastomosis of the right proximal ureter and unified calyces was performed using end-to-end anastomosis, leaving a 6 Fr double-J stent, which was inserted prior to anastomosis (Figure 3). A percutaneous nephrostomy tube and a retroperitoneal drain was placed before wound closure.

The patient had good postoperative recovery without any remarkable event. The retroperitoneal drain was removed on the third day after surgery, and the patient was discharged the following day. Pathological analysis of the excised renal pelvis showed atrophic transitional epithelial cells. Two weeks after surgery, the patient underwent an antegrade pyelogram. The nephrostomy tube was removed after confirming that there was no obstruction or extravasation visible. The double-J stent was removed 3 months after nephrostomy removal. The patient had no complaints, and no hydronephrosis was found from ultrasonography examinations performed at 1 and 9 months after double-J stent removal (Figure 4). Furthermore, urinalysis showed no

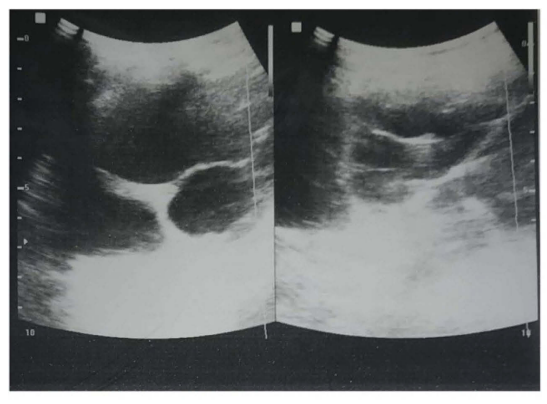

A

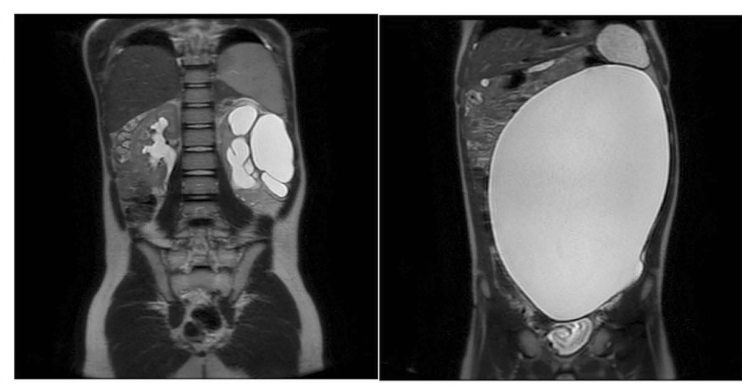

B

Figure I Preoperative (A) ultrasonography and (B) magnetic resonance $T_{2}$-weighted image. 


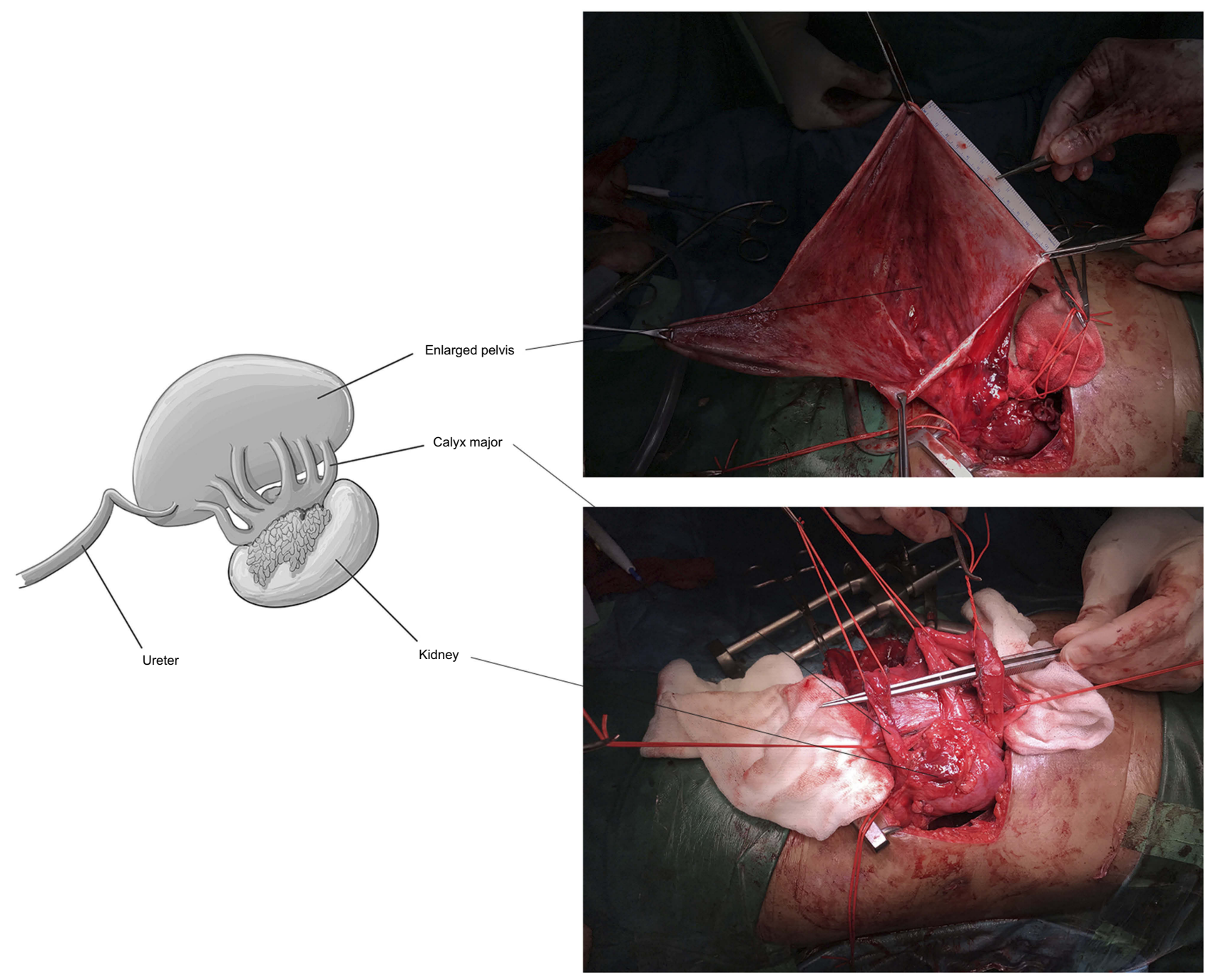

Figure 2 Intraoperative finding of extrarenal calyces and schematic.

infection, and ${ }^{99 \mathrm{~m}} \mathrm{Tc}$ DTPA diuretic renal scan revealed a slight decrease in GFR (20.7 mL/minute).

\section{Discussion}

ERCs are a rare urinary tract anomaly, described as the presence of a major calyx (or calyces) and renal pelvis outside the kidney. The exact incidence of this anomaly is unknown; however, one study reported incidence of $1 \%$ from 300 autopsies. Another report stated that left ERCs is more common than on the right. ${ }^{9}$ The pathogenesis of ERCs is still in question. A number of hypotheses have been proposed regarding their formation, including relatively rapid development of the ureteric bud or relatively slow development of metanephric tissue. ${ }^{10}$ Moreover, ERCs are also associated with other anomalies, such as UPJO, horseshoe kidney, ectopic kidney, and renal dysplasia. ${ }^{2-8}$ In this case, we found ERCs on the left kidney that were accompanied by UPJO.
The finding of ERCs is often incidental and made intraoperatively, surprising the surgeon. There has been no report on the most accurate diagnostic tool to date. The clinical presentation of ERCs varies widely, ranging from asymptomatic to urinary tract infection, presence of an abdominal mass, hydronephrosis, or even spontaneous renal pelvis rupture. ${ }^{4,7,8,11}$ Hydronephrosis or abdominal mass is the most common finding, which was also found in this case. The literature suggests that ultrasonography in ERC cases often reveals hydronephrotic kidney, which appears to be accompanied by multicystic kidney disease. $^{7,9,12}$ This feature was also found in our patient. It is thus important to detect such ultrasonography images, as they are expected to increase the possibility of patients having ERCs. Moreover, UPJO appearance on radiological findings has a nonclassical appearance, and distorted calyces should also raise suspicion. ${ }^{6}$ 

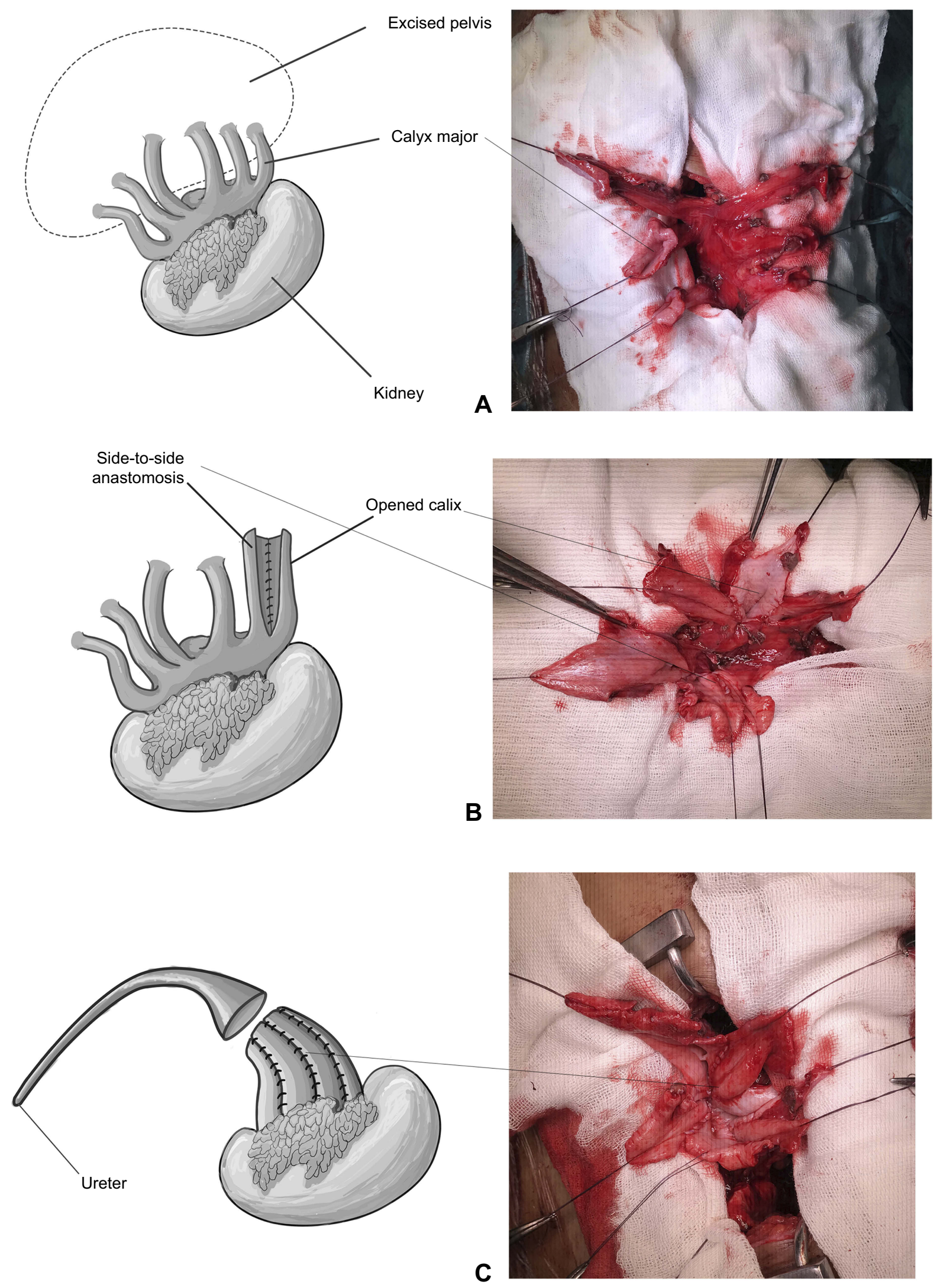

Figure 3 (A) Major calyces after removal of dilated renal pelvis and schematic; (B) side-to-side anastomosis between major calyx and schematic; (C) calyx unification and schematic. 


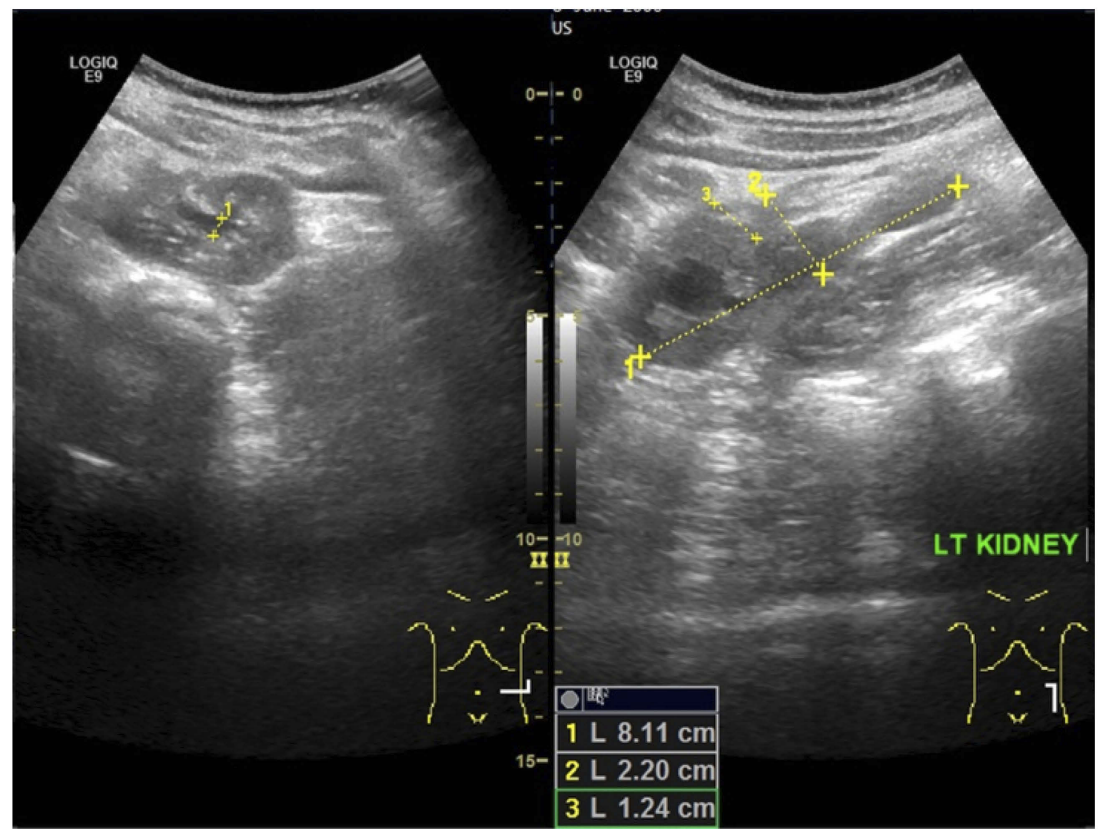

Figure 4 Ultrasonography examination performed 9 months after double-J stent removal.

There is no definitive guideline regarding the treatment of patient with ERCs associated with UPJO. The choice of treatment should be based on residual kidney function. In dysfunctional kidneys, nephroureterectomy should be the treatment of choice. ${ }^{9}$ In functioning kidneys, the kidney should be preserved. There have been at least two successful reports on pyeloplasty, either open surgery or laparoscopically, as a management of choice to treat ERCs associated with UPJO$^{7,8}$

In this report, the patient had no complaints, and ultrasonography examination showed no hydronephrosis after renal pelvis reconstruction using calyx-unification surgery. Even though the patient experience a decrease in GFR, this could have been caused by the surgery, which was performed 4 months after the initial ${ }^{99 \mathrm{~m}} \mathrm{Tc}$ DTPA diuretic renal scan. Overall, our management of this case could be considered a safe and successful novel approach to treat ERCs associated with UPJO.

\section{Conclusion}

We conclude that in a patient with symptomatic ERCs coinciding with UPJO and with good residual renal function, dismembered pyeloplasty could be the first choice of treatment if part of thepyelum can be maintained. However, in the case of a very large renal pelvis or when a portion of the renal pelvis is difficult to maintainednd renal pelvis excision cannot be avoided, the use of renal pelvis reconstruction using calyx unification could be opted for as the treatment of choice.

\section{Consent statement}

We obtained written informed consent from the patient's parents for all the anonymized information to be published.

\section{Ethical approval}

Approval was given by the Cipto Mangunkusumo Hopsital Ethics Committee for reporting this case.

\section{Disclosure}

The authors report no conflicts of interest in this work.

\section{References}

1. Eisendrath D. Hydronephrosis in kidney with extrarenal calyces. J Urol. 1925;13:51-56. doi:10.1016/S0022-5347(17)73548-3

2. Mohanty C, Ray B, Samaratunga U, Horseshoe SG. Kidney with extrarenal calyces - a case report. J Anat Soc India. 2002;51(1):57-58.

3. Suwannakhan A, Meemon K. Horseshoe kidney with extrarenal calyces and malformed renal vessels. Eur J Anat. 2016;20(4):355-359.

4. Singh V, Gupta DK, Pandey M, Kumar V. Congenital right sided ureteropelvic junction obstruction in right crossed fused ectopia with extrarenal calyces masquerading as massive retroperitoneal urinoma in a case of blunt trauma abdomen: a diagnostic enigma and. African J Urol. 2013;19(4):194-197. doi:10.1016/j.afju.2013.07.004

5. Taha SA, Hashish M, Zahrani A. Renal dysplasia with extrarenal calyces. Saudi Med J. 2006;27(3):392-394.

6. Raghunath B, Narendra B, Gowrishankar B, Ramesh S. Extrarenal calyces associated with pelviureteric junction obstruction: a case report of a rare anomaly. J Indian Assoc Pediatr Surg. 2012;17(3):124-125.

7. Ransford G, Young E, Castellan M, Labbie A. Renal pelvis rupture in a kidney with ureteropelvic junction obstruction and extrarenal calyces. $J$ Pediatr Urol. 2013;9(3):127-130. doi:10.1016/j. jpurol.2012.12.018 
8. Wadhwa P, Hemal AK. Case report: transmesocolic laparoscopic reconstruction of ureteropelvic junction obstruction in pelvic kidney associated with extrarenal calices. J Endourol. 2006;20(3):188-190. doi:10.1089/end.2006.20.560

9. González Maldonado AA, Manzo Pérez G, Vanzzini Guerrero MA, Marte Aracena EM, Manzo Pérez BO, Sánchez López HM. Extrarenal calyces as a cause of non-functional kidney in a child: case report. Urol Case Rep. 2018;17:56-58. doi:10.1016/j. eucr.2017.11.004
10. Gupta N, Bhandari M. Extrarenal pelvis and calyces. In: Challenging and Rare Case in Urology. 1st ed. New Delhi: Jaypee Brothers Medical Publishers; 2014:29-31.

11. Gupta T, Goyal SK, Aggarwal A, Sahni D, Mandal AK. Extrarenal calyces: a rare renal congenital anomaly. Surg Radiol Anat. 2015;37(4):407-410.

12. Rajendran S, Cho A, Mishra P, Cherian A. Hydronephrotic kidney with multiple extra-renal calyces. Ann R Coll Surg Engl. 2017;99(8): e219-e220. doi:10.1308/rcsann.2016.0287

\section{Publish your work in this journal}

Research and Reports in Urology is an international, peer-reviewed, open access journal publishing original research, reports, editorials, reviews and commentaries on all aspects of adult and pediatric urology in the clinic and laboratory including the following topics: Pathology, pathophysiology of urological disease; Investigation and treatment of urological disease; Pharmacology of drugs used for the treatment of urological disease. The manuscript management system is completely online and includes a very quick and fair peer-review system, which is all easy to use. Visit http://www.dovepress.com/ testimonials.php to read real quotes from published authors 